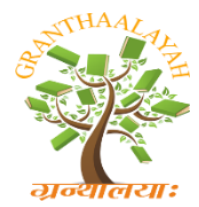
\author{
GRANTHAALAYAH \\ A knowledge Repository
}

INTERNATIONAL JOURNAL OF RESEARCH - 圆圆

Science

\title{
THE RELATIONSHIP BETWEEN INSULIN RESISTANCE AND FA- TIGUE SYMPTOM IN FIBROMYALGIA SYNDROME
}

\author{
Türkan Turgay ${ }^{* 1}$, Zekiye İpek Katirci Kirmaci ${ }^{2}$, Pınar Günel Karadeniz ${ }^{3}$, Mehmet \\ Baştemir $^{4}$ \\ ${ }^{1}$ Sanko University, Faculty of Medicine, SANKO University, Faculty of Medicine, \\ Department of Physical Medicine and Rehabilitation, Turkey \\ ${ }^{2}$ Sanko University, Faculty of Health Science, Department of Physiotherapy and \\ Rehabilitation, Turkey \\ ${ }^{3}$ Sanko University, Faculty of Medicine, Department of Biostatistics, Turkey \\ ${ }^{4}$ Sanko University, Faculty of Health Science, Department of Nutrition and Dietetics, \\ Turkey
}

\begin{abstract}
The aim of this study is to examine the relationship between insulin resistance and fatigue in fibromyalgia. A total of 72 patients (4 males, 68 females) with fibromyalgia (FM) was investigated. Patients were divided into two groups by insulin resistance (IR), group 1; IR- (n=49), and group 2; IR+ ( $\mathrm{n}=23)$. IR was determined using homeostasis model assessment (HOMA) formula. All subjects were evaluated with serum $25(\mathrm{OH})$ D vitamin levels. The severity of the disease was measured with the Fibromyalgia Impact Questionnaire (FIQ); the level of fatigue was evaluated with Fatigue Severity Scale (FSS); and the pain level was evaluated with Visual Analog Scale (VAS). There were significant differences between the FIQ and FSS mean scores of two groups $(\mathrm{IR}+$ and IR-), $(\mathrm{p}=0.031, \mathrm{p}=0.005)$. There were no significant differences between two groups in respect to VAS and serum $25(\mathrm{OH}) \mathrm{D}$ vitamin levels $(\mathrm{p}=0.789, \mathrm{p}=0.875)$. There was no significant correlation be-tween vitamin D and FSS (IR+, r=-0.263, p=0.225; IR-, $r=0.112, p=0.443$ ), and also vitamin $\mathrm{D}$ and FIQ (IR+, $\mathrm{r}=-0.103, \mathrm{p}=0.641$; IR-, $\mathrm{r}=0.050, \mathrm{p}=0.733$ ) in both groups. There was no relationship be-tween insulin resistance and fatigue symptom in fibromyalgia patients, but it was observed that the severity of fatigue was higher in the group with insulin resistance.
\end{abstract}

Keywords: Fatigue; Fibromyalgia; Insulin Resistance.

Cite This Article: Türkan Turgay, Zekiye İpek Katirci Kirmaci, Pınar Günel Karadeniz, and Mehmet Baştemir. (2020). "THE RELATIONSHIP BETWEEN INSULIN RESISTANCE AND FA-TIGUE SYMPTOM IN FIBROMYALGIA SYNDROME.” International Journal of Research - Granthaalayah, 8(3), 271-278. 10.29121/granthaalayah.v8.i3.2020.157. 


\section{Introduction}

Fibromyalgia (FM) is a non-articular chronic disease characterized by widespread musculoskeletal pain, morning stiffness and excessive tenderness at specific anatomical points. In addition to pain, this condition is mostly reported with fatigue, disruption of sleep, impaired cognition, depression, irritable bowel disease with unknown etiology. The disease is more frequent in female adults with a prevalence of $2.4 \%$ and $6.8 \%(1)$.

Fatigue is a complicated and annoying symptom that is obstinately permanent for both patients with fibromyalgia and their physicians as shown by substantially recent studies (2). Fatigue is a frequent complaint in FM at least as much as pain. In this condition patients use several manifestations to describe their experience of fatigue such as 'I am tired', 'I have no energy' and 'I feel worn out' or 'I am exhausted'. It has been pointed out that there is a concurrence among patients that FM-associated fatigue is more intense than normal fatigue (3).

The relationship between chronic pain and metabolic disorders is not still well known. But FM is seen prevalently between 40-70\% people who are overweight or obese (4), people who are diabetes mellitus in overweight or obese, (5) and also who has metabolic syndrome (6).

Previous studies have shown that severe conditions predispose to the development of IR such as obesity, physical inactivity (7), Alzheimer (8), Multiple Sclerosis (9) and Parkinson Disease (10). All these studies have suggested that biological changes in glucose and insulin may be associated with signs of fatigue.

It has been shown that the presence of insulin resistance (IR) increases the chance of falling in patients with fibromyalgia 1.8 times and it is suggested that symptoms of FM may influence muscle strength (11). In a similar study, it is reported that IR is a significant parameter for memory issues in FM patients (12).

The relationship between metabolic disorders and fatigue in FM patients have been studied less frequently than the other FM-related symptoms. Therefore, we aimed to establish the pathogenetic relationship between fatigue severity and IR in patients with FM which may lead to new approaches in the management of this disorder.

\section{Materials and Methods}

A total of 72 patients with FM who applied to Physical Medicine and Rehabilitation and Endocrinology outpatient clinics between Sep 2018-Feb 2019 at SANKO University Sani Konukoğlu Research and Practice Hospital were included in this retrospective study. The study protocol was approved by the University Ethics Committee for Clinical Trials (Decision No: 2018/08-07; Date: 24 July 2018). Patients newly diagnosed with fibromyalgia according to the American College of Rheumatology (ACR) diagnostic criteria were included in this study (13). Age, gender, height and weight were recorded according to the data obtained from the patients' files. BMI was calculated as weight in kilograms divided by height in meters squared $\left(\mathrm{kg} / \mathrm{m}^{2}\right)$. We obtained basal plasma glucose and insulin levels of patients from their files. IR was calculated by the homeostasis model assessment HOMA formula (HOMA formula: basal plasma glucose 
$(\mathrm{mg} / \mathrm{dl}) \times$ basal plasma insulin (UI/ml/405). Finally, it was accepted that patients with more than 2.5 values had insulin resistance. We also examined the vitamin D levels of the patients who were admitted with fatigue, weakness and exhaustion. Serum $25(\mathrm{OH}) \mathrm{D}$ vitamin $(\mathrm{ng} / \mathrm{ml})$ levels were reported. We used $30 \mathrm{ng} / \mathrm{ml}$ as a distinctive marker in this study (absence below $29 \mathrm{ng} / \mathrm{ml}$, normal value $30 \mathrm{ng} / \mathrm{ml}$ and above) (14).

Patients were classified into two groups, based on the presence of insulin resistance:

Group 1; IR-: 49 FM patients without insulin resistance

Group 2; IR+: 23 FM patients with insulin resistance

We excluded patients with a history of thyroid disease, infection, malignancy, neuromuscular diseases, severe cardiovascular and pulmonary diseases, chronic liver and kidney disease, severe anemia, pregnancy, patients with severe osteoarthritis and those who used drugs effective in the central nervous system such as antiepileptic, antidepressant, pregabaline and opioids in order to eliminate reasons that may cause of fatigue. We also did not include patients with diabetes and body mass index (BMI)> 25 taking into account impaired IR in diabetes mellitus and obesity.

We used some health related quality of life instruments to evaluate symptoms of FM. Severity of FM is measured with the Fibromyalgia Impact Questionnaire (FIQ) which measures physical functioning, work status (off days of work and job difficulty), mood, morning tiredness, pain, stiffness, fatigue, and well-being over the past week. Scores range from 0 to 100 with a higher value indicating a greater impact of the disorder (15). Fatigue is evaluated by using The Fatigue Severity Scale (FSS) which is measures how fatigue affects motivation, exercise, physical functioning, carrying duties, interfering with work, family and social life scoring each indicator on 7-point Likert scale, ( $1=$ I totally disagree, to $7=$ I totally agree $)$. A total score of 36 or higher indicates severe fatigue (16). Generalized pain experienced in the last week assessed by using Visual Analog Scale (VAS), on a 0 (no pain) to 10 (worst pain) rating scale. FIQ, FSS and VAS parameters were obtained from the baseline records of patients.

The study was conducted in accordance with the principles of the Declaration of Helsinki.

\section{Statistical Analysis}

IBM SPSS Statistics 23 package program was used for statistical analyses (SPSS Inc., Chicago, IL, USA). Descriptive statistics were given as mean \pm standard deviation, median (minimummaximum), frequency and percentage. Normality of data was evaluated with Shapiro-Wilk test. Independent samples t-test or Mann-Whitney $U$ test was used for comparison of two groups according to distribution of data. Pearson's correlation coefficient was used for assessing relationships between continuous variables. For all analyses $\mathrm{p}<0.05$ was considered statistically significant.

\section{Results and Discussions}

In this study 72 FM patients (4 males, 68 females) were enrolled. Totally 23 patients (31.9\%) had insulin resistance. Demographic characteristics of the groups are presented in Table 1. Age, height, weight and BMI were similar in both groups ( $\mathrm{p}>0.05)$. 
Significant differences between the groups were observed with the results of the FIQ and FSS $(\mathrm{p}<0.05)$. However, no significant difference was detected between groups for the results of VAS and serum $25(\mathrm{OH}) \mathrm{D}$ vitamin $(\mathrm{p}>0.05)$. Vitamin D levels were below $30 \mathrm{ng} / \mathrm{ml}$ in both groups (Table 1).

There was no significant correlation between vitamin D and FSS (IR+, r=-0.263, p=0.225; IR-, $\mathrm{r}=0.112, \mathrm{p}=0.443$, respectively), and also vitamin D and FIQ (IR+, $\mathrm{r}=-0.103$, IR-, $\mathrm{p}=0.641$; $\mathrm{r}=0.050, \mathrm{p}=0.733$, respectively) in both groups.

Table 1: Characteristics of study groups

\begin{tabular}{|l|c|c|c|}
\hline Variables & $\begin{array}{c}\text { Group IR- (n=49) } \\
\text { Mean } \pm \text { SD } \\
\text { or } \\
\text { Median }(\mathbf{I Q R})\end{array}$ & $\begin{array}{c}\text { Group IR+ }(\mathbf{n = 2 3}) \\
\text { Mean } \pm \text { SD } \\
\text { or } \\
\text { Median }(\mathbf{I Q R})\end{array}$ & $\mathbf{p}$ \\
\hline Age & $36.35 \pm 10.26$ & $37.13 \pm 11.56$ & $0.773 \mathrm{a}$ \\
\hline Height $(\mathrm{cm})$ & $162.71 \pm 5.67$ & $164.70 \pm 7.10$ & $0.207 \mathrm{a}$ \\
\hline Weight $(\mathrm{kg})$ & $60.14 \pm 5.96$ & $62.04 \pm 7.62$ & $0.253 \mathrm{a}$ \\
\hline BMI $(\mathrm{kg} / \mathrm{m} 2)$ & $22.45 \pm 1.82$ & $22.91 \pm 1.73$ & $0.314 \mathrm{a}$ \\
\hline FIQ & $59.22 \pm 11.55$ & $65.47 \pm 10.48$ & $0.031 \mathrm{a}$ \\
\hline FSS & $5.49 \pm 0.88$ & $6.06 \pm 0.50$ & $0.005 \mathrm{a}$ \\
\hline VAS & $7.55 \pm 1.66$ & $7.44 \pm 1.83$ & $0.789 \mathrm{a}$ \\
\hline Serum $25(\mathrm{OH})$ & $14.50(15.65)$ & $14.20(12.80)$ & $0.875 \mathrm{~b}$ \\
\hline
\end{tabular}

andependent samples t-test, ' ${ }^{\mathrm{b}}$ Mann-Whitney U test, IR-: without Insulin Resistance, IR+: with Insulin Resistance; SD: Standard Deviation; IQR: Interquartile Range; BMI: Body Mass Index; FIQ: Fibromyalgia Impact Questionnaire; FSS: Fatigue Severity Scale; VAS: Visual Analog Scale

\section{Conclusions and Recommendations}

In the present study, we investigated the prevalence of IR in 72 female patients with normoglycemic FM. Both groups had BMI $<25$ in patients who did not have diabetes and metabolic syndrome. This study revealed that FM patients with IR+ had higher prevalence of disease activity which was assessed by FIQ and fatigue symptoms than the patients with IR-. Both groups showed no significant difference in VAS parameters and vitamin D levels.

FM is one of the main causes of diminished physical functioning along with fatigue (17). Individuals with FM revealed that the intensity of fatigue is often more severe and troublesome than pain. Indeed, studies have shown that chronic pain (both due to central and peripheral sensitization), sleep disturbance, negative mood, anxious affect or combination of all these symptoms are related with fatigue. Differences such as medical use, physical activity, antiepileptic, life style changes and educational level may affect the symptoms of disease severity and fatigue in patients with FM (18). 
Bonora and Bosco et al. showed that remarkable number of subjects was IR+ without metabolic syndrome in healthy population $(19,20)$. In this study, there is no healthy control group to compare the effect of insulin resistance. The evaluation was only performed on the patients with FM.

Çakit et al. found that obesity have significant negative effects on pain, severity of disease and quality of life in normoweight and overweight patients with FM (21). A similar study has shown that higher BMI levels are associated with clinical findings of FM (22). Although it is not clear how obesity affects FM symptoms, epidemiological data indicates that fibromyalgia patients compared with healthy patients are commonly obese (40\%) and overweight's (30\%) in multiple studies. Sleep disorders, depression, anxiety, thyroid gland dysfunction, GH/IGF-1 axis disorder, the disruption of endogenous opioid system may explain why obesity develops in FM. The relationship between obesity (by insulin resistance) and chronic pain is very complex, besides it is possible to explain whether exercise can decrease pain and/or recover metabolic changes due to obesity. Studies have shown that insulin resistance is more common in patients who are obese or overweight fibromyalgia than those with normal weight (4). These results are explained by the connection between reduced physical activities in obese or sedentary individuals. Fibromyalgia patients tend to inactivity due to fatigue. Most fibromyalgia patients describe that they couldn't even do daily activities. The FIQ is a useful tool to assess physical activity in patients with FM. On the other hand, the increase in FIQ scores in the IR+ group seems to be consistent with fatigue symptoms in this study. Since the individuals with FM are frequently overweight or obese, the development of IR may be inevitable. Unlike previous studies, the striking point of our study was that we did not include BMI> 25 patients in this study to eliminate the expected fatigue symptoms in clinically obese and overweight individuals.

IR has been related to an increased risk for falls in the FM patients with chronic pain. In fact, there are evidence that acute pain can lead to insulin resistance and insulin metabolism disorder through the neural elements mediated mechanism, but evidences exploring the link between chronic pain and metabolic disturbances is still unknown. Moreover, insulin resistance results in changes in the metabolism of muscles by stimulating the loss of lean muscle mass (23). These findings may attend to the presence of the fatigue and low aerobic exercise capacity in patients with IR.

Similar to our study, Fava et al. correlated the "memory deficit' seen in fibromyalgia with insulin resistance (12). It is known that there is a close relationship between fatigue, stress, memory deficit and depression. Therefore, according to our results IR may be a potential factor affecting on fatigue due to chronic stress through noradrenergic responses to induced hyperinsulinemia. Similarly, in a very recent study, Pappolla et al. reported that HbA1c levels, an indicator for impaired glucose metabolism characterized by IR, were significantly increased in FM patients compared to the control group (24). These studies suggest that insulin resistance may be associated with the most drastic symptoms of the disease, such as fatigue related pain.

It is well known that insulin resistance is a key feature of metabolic syndrome (MS). IR has been shown as the major supporter link between physical inactivity and MS (25). Dysregulation of hypothalamic pituitary axis with chronic pain and fatigue induced hypocortisolism are associated development of IR and metabolic syndrome, and also FM (26). 
In our study IR was present in $31 \%$ of all patients. However, this result cannot be the full explanation whether insulin resistance is associated with fatigue symptoms in FM when it is considered the other factors such as vitamin D levels, exercise, diet and lifestyle. Because of these factors, we also reviewed the vitamin D levels believed to contribute to fatigue in subjects.

In our study, there was no correlation with vitamin $\mathrm{D}$, fatigue and fibromyalgia impact scale in both IR+ and IR-groups. There are many studies evaluating vitamin D levels in fibromyalgia and chronic musculoskeletal diseases, but the results are contradictory. Özcan et al. showed that the levels of fatigue in patients with vitamin D levels below $20 \mathrm{ng} / \mathrm{mL}$ increased dramatically (27). Roy et al. found out vitamin D deficiency among 77.2\% of patients who do not have severe chronic disease (28). The same authors reported that vitamin D replacement significantly reduced fatigue levels. In another study, including 130 patients diagnosed with FM, Kasapoğlu et al. found no significant association between low vitamin D levels and fatigue in both FM group and healthy control groups (29).

Environmental factors are as a potential contributor to insulin resistance related fatigue. It is well known that trained subjects and those with regular physical activity as a lifestyle have high levels of insulin sensitivity/insulin action. But patients tend to be sedentary behaviours and physical inactivity in FM. In a recent study Bjersing et al investigated that how metabolic factors correlate to the effects of resistance training and found that significant reduction in insulin-like growth factor 1 and leptin levels, improvement in pain, fatigue and muscle strength in lean patients with FM (30). In a similar study Busch et al showed that aerobic and strength training improves physical fitness and decrease fibromyalgia symptoms (31).

The current study has some limitations. There was no control group including individuals without FM therefore we were unable to compare the fatigue severity between FM and controls. In addition, the study consisted mostly female patients. We believe that further prospective controlled studies are necessary and it can be provided large scale studies including more male patients on the relationship between IR, fatigue and severity of FM symptoms. Future studies should aim to determine IR+ in patients with FM and to investigate how fatigue will respond to rehabilitation interventions to enhance aerobic capacity and individualized aerobic exercises. These results may vary in studies conducted in populations with different characteristics such as genetic factors, psychological variables, exercise or dietary habits.

In conclusion, our results support the need to consider that IR may be a risk factor of fatigue severity in patients with FM and if confirmed, it might be an assessment of fatigue symptoms in FM patients. Thus, fibromyalgia patients may be administered a drug therapy or an exercise program targeting insulin resistance, which significantly reduces the symptoms of fatigue.

\section{Declaration of Conflict of Interest}

The authors declared no conflicts of interest with respect to the authorship and/or publication of this article. 


\section{Funding}

The authors received no financial support for the research and/or authorship of this article.

\section{References}

[1] Marques AP, Santo ASDE, Berssaneti AA, Matsutani LA, Yuan SLK. Prevalence of fibromyalgia: literature review update. Rev Bras Reumatol Engl Ed. 2017:4; 356-363.

[2] Walitt B, Fitzcharles MA, Hassett AL, Katz RS, Häuser W, Wolfe F. The longitudinal outcome of fibromyalgia: a study of 1555 patients. J Rheumatol. 2011:38; 2238-2246.

[3] Humphrey L, Arbuckle R, Mease P, Williams DA, Samsoe BD, Gilbert C. Fatigue in fibromyalgia: a conceptual model informed by patient interviews. BMC Musculoskelet Disord. 2010:11(9); 216.

[4] Okifuji A, Bradshaw DH, Olson C. Evaluating obesity in fibromyalgia: neuroendocrine biomarkers, symptoms, and functions. Clin Rheumatol. 2009:28(4); 475-478.

[5] Yanmaz MN, Mert M, Korkmaz M. The prevalence of fibromyalgia syndrome in a group of patients with diabetes mellitus. Rheumatol Int. 2012:32(4); 871-874.

[6] Loevinger BL, Muller D, Alonso C, Coe CL. Metabolic syndrome in women with chronic pain. Metabolism. 2007:56(1);87-93.

[7] Amati F, Dubé JJ, Coen PM, Stefanovic-Racic M, Toledo FG, Goodpaster BH. Physical inactivity and obesity underlie the insulin resistance of aging. Diabetes care. 2009:32(8):1547-9.

[8] Dineley KT, Jahrling JB, Denner L. Insulin Resistance in Alzheimer's Disease. Neurobiol Dis. 2014:72(12);92-103.

[9] Oliveira SR, Simão AN, Kallaur AP, de Almeida ER, Morimoto HK, Lopes J et al. Disability in patients with multiple sclerosis: influence of insulin resistance, adiposity, and oxidative stress. Nutrition. 2014: 30(3); 268-73.

[10] Athauda D, Foltynie T. Insulin resistance and Parkinson's disease: A new target for disease modification? Prog Neurobiol. 2016:10-11(145-146); 98-120.

[11] Góes SM, Stefanello JM, Homann D, Lodovico A, Hubley-Kozey CL, Rodacki AL. Torque and muscle activation impairment along with insulin resistance are associated with falls in women with fibromyalgia. J Strength Cond Res. 2016:30(11); 3155-3164.

[12] Fava A, Plastino M, Cristiano D, Spanò A, Cristofaro S, Opipari C et al. Insulin resistance possible risk factor for cognitive impairment in fibromialgic patients. Metab Brain Dis. Metab Brain Dis. 2013:28;619.

[13] Wolfe F, Clauw DJ, Fitzcharles MA, Goldenberg DL, Katz RS, Mease P et al. The American College of Rheumatology preliminary diagnostic criteria for fibromyalgia and measurement of symptom severity. Arthritis Care \& Research. 2010:62(5); 600-610.

[14] Beckmann Y, Türe S, Duman SU. Vitamin D deficiency and its association with fatigue and quality of life in multiple sclerosis patients. EPMA Journal. 2020:11; 65-72.

[15] Ediz L, Hiz O, Toprak M, Tekeoğlu I, Ercan S. The validity and reliability of the Turkish version of the revised fibromyalgia impact questionnaire. Clin Rheumatol. 2011:30(3); 339-46.

[16] Gencay Can A, Can SS. Validation of the Turkish version of the fatigue severity scale in patients with fibromyalgia. Rheumatol Int. 2012:32(1); 27-31.

[17] Schweiger V, Del Balzo G, Raniero D, De Leo D, Martini A, Sarzi-Puttini P et al. Current trends in disability claims due to fibromyalgia syndrome. Clin Exp Rheumatol. 2017:35; 105(3); 119-126.

[18] Bartley EJ, Robinson ME, Staud R. Pain and fatigue variability patterns distinguish subgroups of fibromyalgia patients. J Pain. 2018:19(4):372-381.

[19] Bonora E, Kiechl S, Willeit J, Oberhollenzer F, Egger G, Targher G et al. Prevalence of insulin resistance in metabolic disorders: the Bruneck study. Diabetes 1998:47; 1643-1649. 
[20] Bosco D, Costa R, Plastino M, Branca D, Cotronei P, Sperlì T et al. Glucose metabolism in the idiopathic blepharoptosis: utility of the oral glucose tolerance test (OGTT) and of the insulin resistance index. J Neurol. 2009:284; 24-28.

[21] Çakıt MO, Çakıt BD, Genç H, Pervane Vural S, Erdem HR, Saraçoğlu M et al. The association of skinfold anthropometric measures, body composition and disease severity in obese and non-obese fibromyalgia patients: A cross-sectional study. Arch Rheumatol. 2017:5; 33 (1); 59-65.

[22] Koçyiğit BF, Okyay RA. The relationship between body mass index and pain, disease activity, depression and anxiety in women with fibromyalgia. Peer J. 2018:6; 4917.

[23] Ursini F, Naty S, Grembiale RD. Fibromyalgia and obesity: the hidden link. Rheumatol Int. 2011:31(11); 1403-8.

[24] Pappolla MA, Manchikanti L, Andersen CR, Greig NH, Ahmed F, Fang X et al. Is insulin resistance the cause of fibromyalgia? A preliminary report. PLoS ONE. 2019;14(5): e0216079.

[25] Fukushima Y, Kurose S, Shinno H, Cao Thu H, Takao N, Tsutsumi H et al. Importance of lean muscle maintenance to improve insulin resistance by body weight reduction in female patients with obesity. Diabetes Metab J. 2016:40(2); 147-53.

[26] Roberts CK, Hevener AL, Barnard RJ. Metabolic syndrome and insulin resistance: underlying causes and modification by exercise training. Compr Physiol. 2013:3(1); 1-58.

[27] Özcan DS, Öken O, Aras M, Köseoğlu BF. Vitamin D Levels in Women with Fibromyalgia and Relationship between Pain, Depression, and Sleep. Turk J Phys Med Rehab 2014:60; 329-34.

[28] Mesci E, Mesci N, İçağasığlu A. Association of vitamin D deficiency with fatigue, daytime sleepiness, and physical activity level. Turk J Phys Med Rehab. 2016:62(3); 222-228.

[29] Kasapoğlu Aksoy M, Altan L, Ökmen MB. The relationship between balance and vitamin 25(OH) D in fibromyalgia patients. Mod Rheumatol. 2017:27(5); 868-874.

[30] Bjersing JL, Larsson A, Palstam A, Ernberg M, Bileviciute-Ljungar I, Löfgren M et al. Benefits of resistance exercise in lean women with fibromyalgia: involvement of IGF-1 and leptin. BMC Musculoskelet Disord. 2017;18(1):106.

[31] Busch AJ, Webber SC, Brachaniec M, Bidonde J, Bello-Haas VD, Danyliw AD et al. Exercise therapy for fibromyalgia. Curr Pain Headache Rep. 2011:15(5); 358-67.

*Corresponding author.

E-mail address: turkanharunlar@hotmail.com 